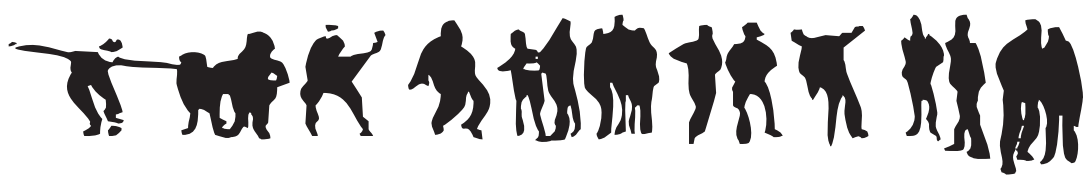

\section{Vers une approche Meta-Design des Learning Games avec le modèle DISC : de la conception à l'analyse des traces d'usage des étudiants par les enseignants}

Mathieu VER MEULEN (LIP6, Sorbonne Université, IMT Lille Douai), Nadine MANDR AN (LIG, Grenoble Alpes Université), Jean-Mar c LAB A T (LIP6, Sorbonne Université), Gaëlle GUIGON (IMT Lille Douai)

RÉSUMÉ • Cet article propose un modèle formel comme partie intégrante d'une approche Meta-Design des Learning Games qui engage fortement les enseignants dans les phases de conception et d'usage. Utilisé comme guide conceptuel dans la phase de conception, le modèle DISC est composé d'éléments déclinables en objets informatiques (artefacts) qui facilitent le travail des enseignants auteurs. Une première expérimentation a permis la création d'un Learning Game en mécanique des fluides. Un travail d'analyse de traces d'usage des étudiants par les enseignants a été facilité par les visualisations proposées et par la structure du modèle DISC. Ce travail a permis une réingénierie du Learning Game.

-MOTS-CLÉS • Meta-Design, jeux sérieux, conception centrée utilisateur, analyse de traces

ABSTRACT • This paper proposes a formal model as part of a meta-design approach to learning games that strongly involves teachers in the design and use phases. Used as a conceptual guide in the design phase, the DISC model is composed of artifacts that ease the teacher-authors work. A first experiment allowed the creation of a learning game in fluid mechanics. An analysis of students' traces of uses has been facilitated by visualizations and the structure of DISC. This work allowed a re-engineering of the learning game.

KEYW ORDS • Meta-design, learning game, User-centered design, traces analysis 


\section{Introduction}

Le terme Learning Game possède plusieurs définitions, en fonction du contexte et des auteurs. Fabricatore (Fabricatore, 2000) en donne une définition qui montre l'ambiguïté du concept:

" a virtual environment and a gaming experience in which the contents that we want to teach can be naturally embedded with some contextual relevance in terms of the game-playing [.... $]^{\Perp}$.

Après un essor important et de nombreux appels à projets publics (par exemple en France), force est de constater le nombre relativement faible de Learning Games produits et l'usage peu important de ceux-ci. Plusieurs facteurs semblent être en cause :

- le manque d'outils et de méthodologies pour les créer, tant pour la conception que pour la production (Mariais et al., 2011);

- le besoin de développements spécifiques dédiés, difficilement réutilisables sans une forte adaptation (liée au choix de l'enseignant) (Marne et Labat, 2014);

- des outils auteurs complexes nécessitant des compétences informatiques (Marfisi-Schottman et al., 2013).

Ces facteurs entraînent, entre autres, un coût élevé pour les Learning Games et ne permettent guère de trouver un modèle économique viable. Par ailleurs, les enseignants de l'enseignement supérieur, même convaincus du potentiel du numérique éducatif dans lequel s'inscrivent les Learning Games, ont des difficultés à se les approprier pour changer leur pédagogie (Egenfeldt-Nielsen, 2004) et ont encore bien plus de difficultés à s'approprier les outils auteurs pour construire leurs propres ressources (Marne, 2014). La conception de ressources pédagogiques par eux-mêmes, ou même simplement leur participation à la conception, serait pourtant probablement un gage de meilleure utilisation de celles-ci, tant les enseignants, en particulier en France, souhaitent rester maîtres des ressources qu'ils utilisent. L'engagement des enseignants à toutes les étapes de la vie d'un Learning Game semble un point important. Des travaux suggèrent un accompagnement de ces derniers lors des phases de

\footnotetext{
${ }^{1}$ Que l'on peut traduire par: un environnement virtuel et une expérience ludique dans lesquels les contenus que l'on souhaite enseigner peuvent être intégrés de façon naturelle, contextuelle et avec pertinence en termes de jeu [...].
} 
conception et d'usage de ces Environnements Informatiques pour l'Apprentissage Humain (EIAH) (Alvarez et al., 2017), d'autres proposent un engagement fort au travers du concept de Meta-Design, que Fischer (Fischer et al., 2004) définit comme suit :

"Meta-design characterizes objectives, techniques, and processes for creating new media and environments allowing "owners of problems" (that is, end users) to act as designers. A fundamental objective of meta-design is to create socio-technical environments that empower users to engage actively in the continuous development of systems rather than being restricted to the use of existing systems ${ }^{2} »$.

Cette approche présente un cadre intéressant en sollicitant fortement les utilisateurs finaux à la fois dans la phase de conception, mais aussi dans la phase d'usage, ce qui leur permettrait de garder un certain contrôle sur les EIAH produits. Notre objectif est de fait de définir des modèles, des méthodes et des outils pour la création de Learning Games qui associent étroitement les enseignants, non seulement dans la phase de conception, mais aussi dans l'usage de ceux-ci. Pour atteindre cet objectif, nous proposons de mettre en place une approche Meta-Design des Learning Games, ce qui requiert de définir un modèle de Learning Game pour faciliter la co-conception de ces derniers, et d'articuler ce dernier avec un outil de visualisation des traces d'usage des étudiants pour permettre l'analyse de celles-ci et donc la réingénierie du Learning Game.

À la suite de cette introduction, nous présentons une étude de l'état de l'art des méthodes et modèles de Learning Games, et les propriétés attendues pour définir un modèle de conception de Learning Games. La partie suivante présente la méthode de conduite de la recherche utilisée, qui a structuré le travail de recherche présenté. Puis, nous proposons un type de Learning Game adapté au contexte de l'enseignement supérieur sur lequel s'appuie le modèle formel DISC décrit dans la quatrième partie de cet article. Nous spécifierons les éléments constitutifs du modèle, ainsi que les relations entre chacun d'entre eux. La partie qui suit traitera de la mise en ouvre de DISC au cours ou tout au long de la création et de

\footnotetext{
${ }^{2}$ Qui peut être traduit par : le Meta-Design définit les objectifs, les techniques et les processus pour créer de nouveaux médias et environnements permettant aux "propriétaires des problèmes " (c'est-à-dire les utilisateurs finaux) d'agir en tant que concepteurs. Un objectif fondamental du Meta-Design est de créer des environnements sociotechniques qui permettent aux utilisateurs de s'engager activement dans le développement en continu des systèmes plutôt que de se limiter à l'utilisation des systèmes existants.
} 
l'usage d'un Learning Game [baptisé Missions à Emosson] dans le domaine de la mécanique des fluides. Cette partie intégrera les retours qualitatifs recueillis auprès des enseignants auteurs. La sixième partie traitera du recueil de traces d'usages des étudiants, de leur analyse par les enseignants, des propositions d'amélioration du Learning Game et de la réingénierie de celui-ci. Enfin, nous proposerons des perspectives de recherche.

\section{2. État de I'art}

Le Meta-Design ne semble pas encore très présent dans la conception des EIAH. Peu d'exemples ont émergé des travaux de recherche, ces derniers s'attaquant principalement à l'une ou à l'autre des phases définies (conception et usage).

Néanmoins, Marne (Marne, 2014) propose une approche Meta-Design des jeux sérieux fondée sur un ensemble de concepts, une méthode de conception et un modèle que nous décrirons plus loin, mais aussi sur la notion d'objets frontières ou Boundary Objects, favorisant l'échange, le partage et la communication entre des acteurs détenteurs d'expertises différentes (Bowker et Star, 2000) et de patrons de conception (ou Design Pattern). Marne propose ainsi des outils utiles dans les phases de conception et d'usage, en insistant sur la réingénierie des jeux sérieux, plus précisément, sur la scénarisation de ceux-ci. Le modèle MoPPLiq définit un cadre permettant d'une part la conception et, d'autre part, l'analyse en vue de faciliter la réingénierie des scénarios des jeux sérieux par les enseignants. Nous reviendrons sur cet outil dans cette partie.

Pour répondre à la problématique de la conception des Learning Games, de nombreux auteurs ont proposé des modèles d'EIAH ou de jeux sérieux souvent génériques, comme Serious Game Conceptual Framework (Yusoff et al., 2009), les 6 facettes du jeu sérieux (Marne et al., 2012), la méthodologie de création de Learning Game utilisée pour l'outil Legadee (Marfisi-Schottman, 2012) ou encore ATMSG (Carvalho et al., 2015). Plus rarement ont été proposés des modèles spécifiques à un type donné, comme le jeu de type Point and Clic avec WEEV (Marchiori et al., 2012) ou MoPPLiq (Marne et Labat, 2014).

Après avoir étudié ces modèles, nous avons extrait des propriétés intéressantes pour notre public cible, les enseignants du supérieur (tableau 1). En reprenant les propriétés des différents modèles étudiés, nous avons choisi de privilégier les propriétés suivantes: identifier clairement la place de l'enseignant et son rôle dans la conception; un 
modèle simple permettant la réingénierie des Learning Games produits ; un modèle décrivant l'ensemble des éléments nécessaires à la conception d'un Learning Game; une conception collaborative fondée sur des composants (activités, mini-jeux, etc.) et sur les approches agiles. Enfin, nous considérons que l'approche narrative est aussi un élément intéressant que l'on souhaite intégrer.

Tableau 1 Propriétés et limites pour différents modèles de Learning Games

\begin{tabular}{|l|l|l|}
\hline Modèle & Propriétés & Limites \\
\hline $\begin{array}{l}\text { SGCF (Yusoffet } \\
\text { al., 2009) }\end{array}$ & $\begin{array}{l}\text { Distingue les étapes de con- } \\
\text { ception et situe les acteurs }\end{array}$ & $\begin{array}{l}\text { Ne précise pas les rôles au } \\
\text { sein du processus }\end{array}$ \\
\hline $\begin{array}{l}\text { 6 facettes } \\
\text { Marne } \text { et al., } \\
\text { 2012) }\end{array}$ & $\begin{array}{l}\text { Propose un cadre de con- } \\
\text { ception souple et identifie 2 } \\
\text { catégories d'expertise }\end{array}$ & $\begin{array}{l}\text { Ne précise pas les objets à } \\
\text { mettre en place et modèle } \\
\text { très général }\end{array}$ \\
\hline $\begin{array}{l}\text { Legadee } \\
\text { (Marfisi- }\end{array}$ & $\begin{array}{l}\text { Articule des mini-jeux dans } \\
\text { un scénario global } \\
\text { 2012) }\end{array}$ & $\begin{array}{l}\text { Propose une approche coo- } \\
\text { pérative en parallèle plutôt } \\
\text { que collaborative }\end{array}$ \\
\hline $\begin{array}{l}\text { ATMSG } \\
\text { (Carvalho } \text { et al., } \\
\text { 2015) }\end{array}$ & $\begin{array}{l}\text { Précise le type d'activités } \\
\text { selon trois catégories: Ga- } \\
\text { ming, Learning, Instructional, } \\
\text { elles-mêmes identifiées par } \\
\text { des actions, des objectifs et } \\
\text { des outils }\end{array}$ & $\begin{array}{l}\text { Propose un modèle théo- } \\
\text { rique assez difficile à appré- } \\
\text { hender par les enseignants }\end{array}$ \\
\hline $\begin{array}{l}\text { WEEV } \\
\text { Marchiori } \text { et al., } \\
\text { 2012) }\end{array}$ & $\begin{array}{l}\text { Propose une approche nar- } \\
\text { rative fondée sur les interac- } \\
\text { tions }\end{array}$ & $\begin{array}{l}\text { Limite la durée du Learning } \\
\text { Game en raison d'une repré- } \\
\text { sentation du scénario com- } \\
\text { plexe et prévu pour un seul } \\
\text { enseignant auteur }\end{array}$ \\
\hline $\begin{array}{l}\text { MoPPLiq } \\
\text { Marne et Labat, } \\
\text { 2014) }\end{array}$ & $\begin{array}{l}\text { Facilite la réingénierie du } \\
\text { scénario }\end{array}$ & $\begin{array}{l}\text { Propose un modèle centré } \\
\text { uniquement sur le scénario }\end{array}$ \\
\hline
\end{tabular}

Ces modèles proposent un cadre capable de décrire les aspects pédagogiques et ludiques d'un Learning Game. Pour autant ils ne précisent pas les modèles informatiques utilisés pour traduire en artefacts la conception de ces EIAH. Or, nous savons par l'expérience d'ingénieur pédagogique de l'un des auteurs de l'article que, sans modèle prédéfini, les enseignants éprouvent des difficultés à penser les objets à mettre en place dans la phase de conception.

De plus, les modèles trop génériques ne facilitent pas la conception et l'usage de Learning Games. Ainsi, nous avons ici pris le parti de proposer 
un modèle formel pour la conception de Learning Game à étapes, autrement dit ceux dont le scénario peut-être fractionné en étapes, qui peuvent être des niveaux, des études de cas ou des exercices de différents types (Marne, 2014). Plus spécifiquement, en nous appuyant sur des travaux antérieurs montrant l'intérêt de la méthode des cas pour l'enseignement supérieur (Marfisi-Schottman et al., 2013), notre modèle permet la conception de Learning Gamebooks ou «jeux sérieux dont vous êtes le héros". Nous définissons un Learning Gamebook comme un Learning Game à étapes, fondé sur un scénario non linéaire, c'est-à-dire un scénario adaptatif en fonction des choix de l'apprenant, des résultats de ses actions ou encore d'éléments aléatoires intégrés, mono-joueur ou multi-joueurs, qui reprend les principes du «Livre dont vous êtes le héros » et des jeux de rôles pédagogiques (Daniau, 2005) en intégrant des études de cas et des problèmes à réaliser par les apprenants-joueurs. On peut rapprocher ce paradigme de celui des Narrative Centered-Learning Environments qui sont définis comme:

"a class of game-based learning environments that contextualize educational content and problem solving with interactive story scenarios " (Rowe et al., 2011). ${ }^{3}$

Le scénario des Learning Gamebooks est donc un élément central, car il favorise les choix de l'apprenant-joueur et lui permet de construire sa propre histoire en fonction de ceux-ci. Nous avons choisi ce type de Learning Game comme base pour définir le modèle DISC.

\section{Une méthode de recherche ancrée sur le terrain:} THEDRE

L'approche méthodologique utilisée pour mener les travaux présentés dans cet article repose sur les travaux de Mandran (Mandran et DupuyChessa, 2017): la méthode de recherche THEDRE pour Traceable Human Experiment Design REsearch.

La méthode THEDRE se situe dans le positionnement épistémologique du constructivisme pragmatique (Avenier, 2009). Elle intègre un processus d'amélioration Plan-Do-Check-Act (PDCA) ou cycle de Deming,

\footnotetext{
${ }^{3}$ Que l'on peut traduire par : une catégorie d'environnements d'apprentissage fondés sur le jeu qui contextualisent le contenu éducatif et la résolution de problèmes au travers de scénarios interactifs sous forme d'histoires.
} 
un outil pour guider l'amélioration d'un processus (Sokovic et al., 2010). Mettre en place ce cycle nécessite de disposer d'indicateurs pour mesurer l'activité, le volume de production et ainsi vérifier que les objectifs définis dans l'action Plan ont été atteints. En particulier, ces indicateurs permettront de déterminer l'intérêt de procéder à une nouvelle itération du cycle.

Un point important pour nous est que THEDRE utilise la démarche centrée utilisateur (DCU) qui intègre l'utilisateur dès le départ de la conception. Les trois phases de la DCU sont l'analyse, la conception et l'évaluation. Mandran propose plutôt les termes Explorer, Co-construire et Évaluer que nous utilisons tout au long de nos travaux, car le terme coconstruction y est particulièrement bien adapté.

La méthode THEDRE se décompose en cinq sous-processus: Planification, Expérimentation, Contrôle, Construction et Décision, et enfin Communication (cf. figure 1). Pour chacun de ces sous-processus, la méthode précise les objectifs et les tâches à atteindre et à réaliser. Elle détaille les livrables à produire et les indicateurs de traçabilité et de qualité associés à ceux-ci. Elle fait intervenir quatre acteurs (figure 1): le chercheur, le développeur, le méthodologue et l'utilisateur. Dans le cadre du travail présenté ici, la contribution du chercheur et du méthodologue est réalisée par la même personne, le doctorant. Le rôle du développeur, quant à lui, est assuré par un ingénieur technopédagogique. Enfin, les utilisateurs sont les enseignants du supérieur, enseignants-chercheurs ou professeurs.

THEDRE fait intervenir des outils activables, comme un média entre l'utilisateur et la connaissance scientifique. Dans notre cas, les outils activables sont :

- Les supports de passation ${ }^{4}$ du modèle de Learning Games (ils dépendent de l'itération du modèle en question); ces supports sont matérialisés par des documents, des cartes mentales ou des tableurs (en général avec le logiciel Excel) ;

- Les Learning Games produits à l'aide du modèle; ce sont des applications $W e b$ intégrées au sein d'une plate-forme de formation (pour nos expérimentations, il s'agit de Moodle).

\footnotetext{
${ }^{4}$ Terme utilisé par Mandran, spécifique à la transmission d'une méthode ou d'un modèle aux utilisateurs en vue de planifier une expérimentation.
} 
Un des points forts de la méthode est d'effectuer un contrôle des données recueillies (sous-processus Contrôle) lors des expérimentations. De fait, le choix de la méthode de production des données est une étape importante de la démarche de recherche pour la recherche en EIAH. Mandran propose un logigramme facilitant cette étape et donnant de la cohérence aux données recueillies. Nous avons largement utilisé celui-ci lors de nos expérimentations.

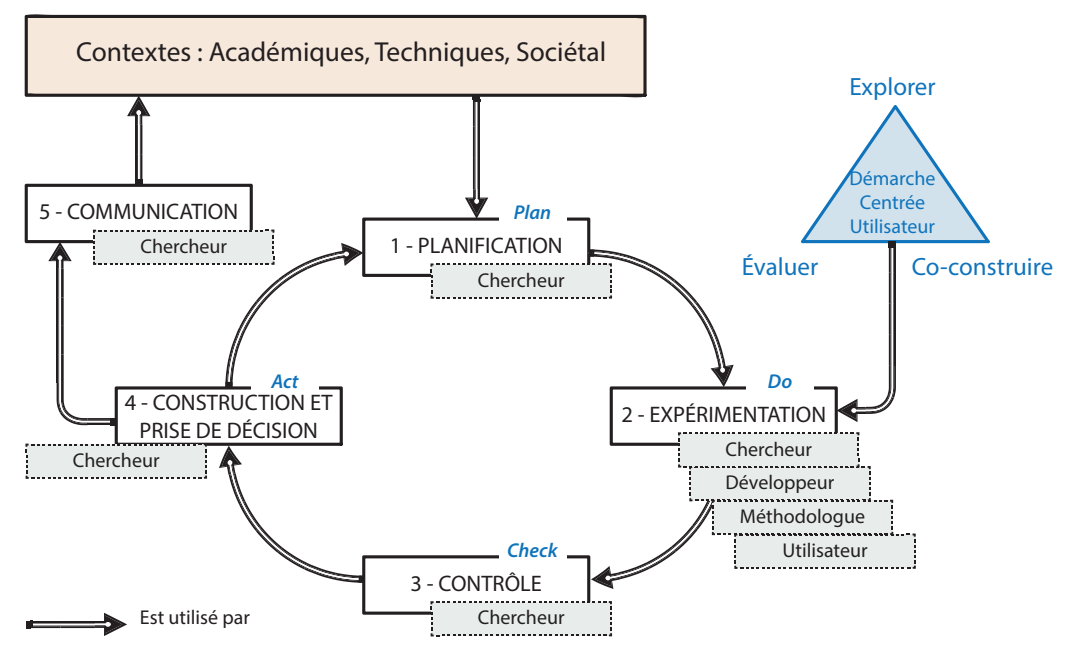

Figure 1 - Méthode THEDRE : 3 contextes, 5 sous-processus, 4 acteurs, Cycle PDCA et Démarche Centrée Utilisateurs (DCU) d'après (Mandran et Dupuy-Chessa, 2017).

La suite de cet article détaille une itération du cycle de recherche de la méthode THEDRE que nous avons mise en œuvre.

\section{Le modèle DISC}

Une première itération du modèle DISC (Vermeulen, 2016) a permis la conception d'un premier Learning Game en février 2016, dont l'usage a été analysé par un suivi des traces d'usages des apprenants (Vermeulen et al., 2016). À la suite de ces travaux, nous avons modifié et précisé le modèle et, 
ainsi, défini une nouvelle version de celui-ci ${ }^{5}$. C'est cette deuxième version qui est décrite dans cet article. Précisons que ce modèle s'intéresse essentiellement à l'objet jeu c'est-à-dire à l'aspect jeu-Game du Learning Game, en parallèle de l'aspect jeu-Play qui concerne l'action de jouer (Sanchez et al., 2015).

Un Learning Game $\mathrm{L}_{\mathrm{g}}$ conçu selon DISC est composé de quatre éléments D, I, S, C :

$$
L_{g}=\{D, I, S, C\}
$$

Pour des raisons de cohérence didactique, nous présenterons dans la suite de cet article ces éléments dans l'ordre suivant: D le modèle du domaine, $\mathrm{S}$ le scénario, I l'ensemble des interactions de $\mathrm{L}_{\mathrm{g}}$ et enfin $\mathrm{C}$ les contraintes d'usage.

Le modèle DISC est constitué de trois structures distinctes, gigognes et hiérarchisées (figure 2): le Learning Game, les étapes et les études de cas.

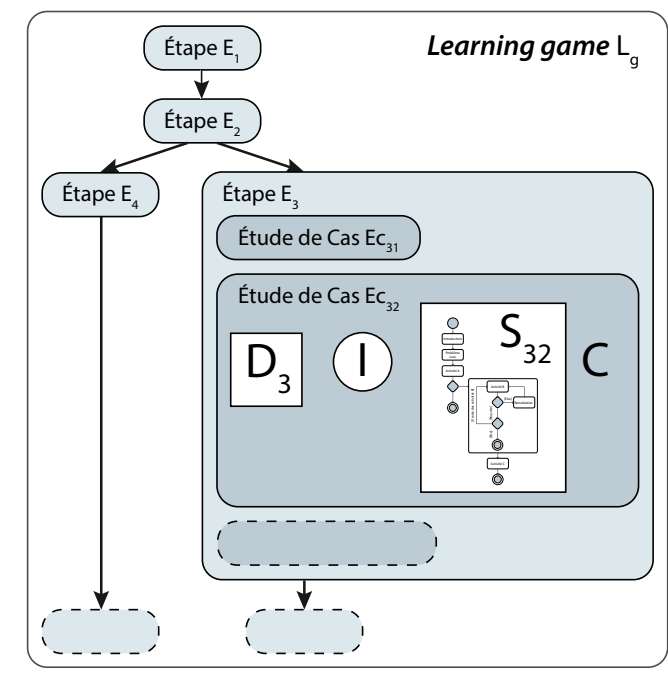

Figure 2 - Structure du modèle DISC

\footnotetext{
${ }^{5}$ Par rapport à la première itération, DISC propose le découpage en étapes de $\mathrm{D}$, la définition et la spécification des études de cas comme briques élémentaires et l'intégration de l'élément $\mathrm{O}$ (Objectifs) comme un sous-élément de $\mathrm{D}$. L'élément $\mathrm{S}$ (scénario) a été décrit en détail avec un focus sur les types d'activités.
} 
Chacun de ces éléments est conçu en étroite collaboration avec l'enseignant auteur du Learning Game, qui est présent dans toutes les phases de la conception. Il est le principal rédacteur-concepteur de ces éléments et de leurs composants. Notre modèle s'adresse en priorité aux enseignants chercheurs scientifiques parce qu'il est préférable de connaitre la notion de graphe pour utiliser notre modèle.

\subsection{Construire le modèle du domaine $D$}

La notion de modèle informatique du domaine n'est pas aisée à comprendre pour les enseignants; ce concept est pourtant indispensable dans la conception d'EIAH: il traduit le recueil de l'expertise des enseignants. Il s'agit ainsi de définir un modèle du domaine compréhensible par les enseignants. Il ne doit pas forcément être exhaustif : il faut restreindre celui-ci au Learning Game à produire et le définir en fonction du public cible du jeu. De plus, ce modèle du domaine est construit par les enseignants en fonction des objectifs pédagogiques à atteindre.

Nous avons choisi de modéliser le domaine $\mathrm{D}$ de $\mathrm{L}_{\mathrm{g}}$ sous la forme d'un graphe orienté dont les nœuds sont les compétences du domaine formant un ensemble noté K. Les arcs de ce graphe traduisent les liens de prérequis entre les compétences du domaine, reprenant ainsi les concepts du CbKST (Reimann et al., 2013), (Melero et al., 2015). Notons Pr l'ensemble de ces liens de prérequis. D est modélisé par le graphe (K, Pr). Ainsi, en reprenant un format simple (le graphe) et aisément représentable, les enseignants peuvent construire le modèle du domaine de manière quasi autonome. Pour autant, la tâche reste difficile dans le cas où les compétences sont en grand nombre.

Pour faciliter la construction du modèle de $\mathrm{D}$, nous proposons de décomposer celui-ci en sous-domaines $\mathrm{D}_{1}, \mathrm{D}_{2}, \ldots, \mathrm{D}_{\mathrm{n}}$. Ce découpage est classiquement réalisé par l'enseignant lors de la construction d'un cours, c'est-à-dire qu'il décompose celui-ci en parties autonomes (chapitres) reliés par des liens de prérequis. Ces sous-domaines sont modélisés par des sousgraphes du graphe de $\mathrm{D}$, respectivement engendrés par des ensembles de compétences $\mathrm{K}_{\mathrm{i}}, \mathrm{K}_{\mathrm{i}} \subset \mathrm{K}$. Les $\mathrm{K}_{\mathrm{i}}$ sont définis par l'enseignant : il définit à la fois les objectifs pédagogiques (l'ensemble des compétences $\mathrm{O}_{\mathrm{i}}$ à atteindre pour chaque chapitre) et les prérequis pour les atteindre (l'ensemble des compétences $\mathrm{P}_{\mathrm{i}}$ ). Ainsi, les $\mathrm{K}_{\mathrm{i}}$ forment des ensembles cohérents de compétences : pour i donné, chaque compétence de $\mathrm{K}_{\mathrm{i}}$ est atteignable par 
un chemin dans le graphe de $\mathrm{D}_{\mathrm{i}}$. Autrement dit, pour toute compétence de $D_{i}$, les prérequis de cette compétence sont des nœuds du graphe de $D_{i}$. Cette décomposition du modèle du domaine selon les différents chapitres facilite la perception de cet élément par les enseignants en rendant l'exercice plus aisé.

Dans le modèle DISC, $\mathrm{L}_{\mathrm{g}}$ contient un ensemble structuré d'étapes $\mathrm{E}_{1}, \mathrm{E}_{2}$, $\ldots, E_{n}$ respectivement associées aux graphes de $D_{1}, D_{2}, \ldots, D_{n}$, faisant respectivement travailler par les apprenants-joueurs les objectifs pédagogiques définis $\mathrm{O}_{1}, \mathrm{O}_{2}, \ldots, \mathrm{O}_{n}$ et ayant respectivement comme prérequis nécessaires pour réaliser ces étapes $\mathrm{P}_{1}, \mathrm{P}_{2}, \ldots, \mathrm{P}_{\mathrm{n}}$. Les $\mathrm{O}_{\mathrm{i}}$ constituent l'ensemble des compétences travaillées au sein de l'étape $\mathrm{E}_{\mathrm{i}}$. Ainsi, pour tout $\mathrm{i} \in[1, \mathrm{n}]$, le graphe de $\mathrm{D}_{\mathrm{i}}$ est le modèle du domaine de l'étape $\mathrm{E}_{\mathrm{i}}$.

\subsection{La construction de $S$ et la décomposition des étapes en études de cas}

La composante $\mathrm{S}$ de $\mathrm{L}_{\mathrm{g}}$ s'exprime de manière différente au sein des trois structures de DISC (figure 2). Au premier niveau, c'est un graphe qui relie les étapes entre elles. Au second niveau, chaque étape est composée d'un ensemble d'études de cas. Des travaux antérieurs ont permis de montrer l'intérêt de la méthode des cas pour la création de Learning Games par les enseignants de l'enseignement supérieur, en particulier à la suite du projet collaboratif Generic-SG (Marfisi-Schottman et al., 2013). Pour tout $\mathrm{i} \in[1, \mathrm{n}]$ l'étape $\mathrm{E}_{\mathrm{i}}$ contient un ensemble de $\mathrm{m}$ études de $\mathrm{cas} \mathrm{Ec}_{\mathrm{i}, 1}$, ..., $\mathrm{Ec}_{\mathrm{i}, \mathrm{m}}\left(\mathrm{m} \in \mathrm{N}^{*}\right.$ et $\mathrm{m}$ dépend de $\left.\mathrm{i}\right)$. $\mathrm{L}_{\mathrm{g}}$ peut ainsi être considéré comme l'ensemble des études de cas $\mathrm{Ec}_{\mathrm{i}, \mathrm{j}}$. Les $\mathrm{Ec}_{\mathrm{i}, \mathrm{j}}$ possèdent un ensemble de prérequis notés $\mathrm{P}_{\mathrm{i}, \mathrm{j}}$ et font travailler par les apprenants-joueurs les objectifs pédagogiques notés $\mathrm{O}_{\mathrm{i}, \mathrm{j}}$.

Nous imposons à l'enseignant que toutes ces études de cas soient indépendantes les unes des autres, ce qui a comme conséquence que l'apprenant peut résoudre les études de cas dans n'importe quel ordre, il n'y a pas de scénario imposé. Cela suppose que pour entrer dans cette étape, l'apprenant maitrise (est censé maitriser) tous les prérequis nécessaires à la résolution de toutes les études de cas. Nous reviendrons plus loin sur cette propriété importante de notre modèle.

Cette décomposition propose aux enseignants de découper la conception en unités de petite taille (les $\mathrm{Ec}_{\mathrm{i}, \mathrm{j}}$ ), ce qui leur semble plus abordable qu'une conception globale. Par ailleurs, cette structuration 
facilite l'intervention de plusieurs enseignants auteurs à condition de respecter le modèle des $\mathrm{Ec}_{\mathrm{i}, \mathrm{j}}$ tel que décrit ci-dessous.

\subsubsection{Le modèle des études de cas $E c_{i, j}$.}

Ainsi, chaque $\mathrm{Ec}_{\mathrm{i}, \mathrm{j}}$ est construite sur le modèle des scénarios utilisateurs (User Stories) issues des approches agiles et des expérimentations d'adaptation de ces approches à la pédagogie (Vermeulen et al., 2015), (Vermeulen et al., 2017). Elles suivent les qualificatifs définis dans l'acronyme P.I.S.T.E. pour Prerequisites, Independent, Small, Testable, Estimable qui est librement inspiré de l'acronyme I.N.V.E.S.T. (Cohn, 2004) issu des approches agiles :

- Prerequisites, les prérequis d'une $\mathrm{Ec}_{\mathrm{i}, \mathrm{j}}, \mathrm{P}_{\mathrm{i}, \mathrm{j}}$ sont connus des apprenantsjoueurs ;

- Independent, les $\mathrm{Ec}_{\mathrm{i}, \mathrm{j}}$ peuvent être réalisées dans l'ordre choisi par l'apprenant-joueur indépendamment de celles qu'il a déjà terminées dans l'étape $\mathrm{E}_{\mathrm{i}}$; ce point essentiel est repris ci-dessous ;

- Small, une $\mathrm{Ec}_{\mathrm{i}, \mathrm{j}}$ doit être suffisamment petite (en terme de ressources pour l'apprenant-joueur);

- Testable, une $\mathrm{Ec}_{\mathrm{i}, \mathrm{j}}$ doit pouvoir être testée ; les objectifs pédagogiques $\mathrm{O}_{\mathrm{i}, \mathrm{j}}$ doivent être atteignables dans un temps court ;

- Estimable, l'apprenant peut initialement estimer les efforts nécessaires pour réaliser une $\mathrm{Ec}_{\mathrm{i}, \mathrm{j}}$.

L'intérêt pour les approches agiles est double dans le modèle DISC. D'une part, elles nous permettent de spécifier les caractéristiques des $\mathrm{Ec}_{\mathrm{i}, \mathrm{j}}$ comme nous l'avons décrit avec l'acronyme P.I.S.T.E. D'autre part, de nombreux outils et méthodes de ce paradigme issu de la gestion de projet en informatique ont déjà été mis en avant dans les méthodes de conception de serious games (Marne, 2014).

\subsubsection{Indépendance des $E c_{i, j}$ et type d'étapes}

L'indépendance des $\mathrm{Ec}_{\mathrm{i}, \mathrm{j}}$ est un point essentiel du modèle DISC. Elle apporte un élément ludique à l'apprenant en lui donnant une liberté visà-vis de l'aspect narratif du scénario global : il peut construire sa propre histoire en suivant l'étude de cas qu'il souhaite au sein d'une étape $\mathrm{E}_{\mathrm{i}}$. De plus, elle garantit la possibilité d'ajouter une étude de cas à $\mathrm{L}_{\mathrm{g}}$ (y compris par un enseignant autre que le concepteur initial de $\mathrm{L}_{\mathrm{g}}$ ) et facilite ainsi la réingénierie du Debriefing Learning Game. Pour qu'il y ait indépendance des $\mathrm{Ec}_{\mathrm{i}, \mathrm{j}}$, il ne doit y avoir aucune relation de précédence ou de prérequis entre deux $\mathrm{Ec}_{\mathrm{i}, \mathrm{j}}$ données. Pour vérifier ce point, il nous faut vérifier 
l'assertion suivante: pour toute étude de cas $\mathrm{Ec}_{\mathrm{i}, \mathrm{j}}$ de $_{\mathrm{i}}$, les prérequis de $\mathrm{Ec}_{\mathrm{i}, \mathrm{i}}$ sont des prérequis de l'étape $\mathrm{E}_{\mathrm{i}}$. Ainsi, quand l'assertion est vérifiée, il est inutile de redéfinir les prérequis pour chaque étude de cas, puisque par hypothèse nous considérons que pour rentrer dans une étape, le joueur doit posséder tous les prérequis pour réaliser n'importe quelle étude de cas de cette étape.

En dialoguant avec les enseignants, nous avons pris conscience qu'ils distinguent deux types d'études de cas $\mathrm{Ec}_{\mathrm{i}, \mathrm{j}}$ en fonction des prérequis et des objectifs de celles-ci. Le premier type dit étude de cas d'acquisition (type 1) est caractérisé par un ensemble $\mathrm{O}_{\mathrm{i}, \mathrm{j}}$ de compétences atteignables en un pas à partir de l'ensemble $\mathrm{P}_{\mathrm{i}, \mathrm{j}}$ dans le graphe $\mathrm{D}_{\mathrm{i}}$. Le second type dit étude de cas de révision (type 2) propose à l'apprenant-joueur de vérifier et de stabiliser un ensemble d'acquis.

Pour les études de cas de type 1 , les objectifs de l'étude de cas sont à un pas d'inférence des prérequis de celle-ci. Dans le cas d'une étude de cas $\mathrm{Ec}_{\mathrm{i}, \mathrm{j}}$ de type 2, celle-ci peut demander plusieurs pas de raisonnement (Vygotsky, 1980).

\subsubsection{Un scénario des $E c_{i, j}$ intégrant des activités}

$\mathrm{Au}$ troisième niveau de notre modèle (figure 2) les $\mathrm{Ec}_{\mathrm{i}, \mathrm{j}} \mathrm{s}^{\prime}$ articulent autour d'un scénario décrit par les enseignants, noté $S_{i, j}, S_{i, j}$ peut être considéré comme un graphe orienté dont les nœuds sont des activités de l'apprenant et dont les arcs traduisent les enchaînements de ces activités choisis librement par les enseignants. Les activités sont en général de difficulté croissante au fur et à mesure de l'avancée dans le graphe de scénario. Du point de vue de l'apprenant, une activité donnée (et donc un nœud dans le graphe du scénario) est déterminée par les choix et actions de l'apprenant dans les activités précédentes (Marne, 2014), (Vermeulen et al., 2016). Les enseignants définissent ainsi un scénario prédictif comme une description à priori du déroulement de la situation de jeu (Pernin et Lejeune, 2004). Les $S_{i, j}$ suivent un modèle de graphe explicité dans la figure 3.

Les $\mathrm{S}_{\mathrm{i}, \mathrm{j}}$ contiennent plusieurs activités ordonnées en fonction de leur difficulté théorique suggérée par l'enseignant. La première activité teste la maitrise des prérequis de l'étude de cas $\mathrm{Ec}_{\mathrm{i}, \mathrm{j}}$ : en cas d'échec de l'apprenant à cette activité, il sort de l'étude de cas. Les suivantes font travailler des compétences incluses dans les $\mathrm{O}_{\mathrm{i}, \mathrm{j}}$ et proposent, en cas d'échec, une remédiation et un nouvel essai. La dernière activité, plus complexe, clôt 
$\mathrm{Ec}_{\mathrm{i}, \mathrm{j}}$ (l'apprenant sort avec un succès ou un échec). Pour chaque activité, les résultats sont enregistrés et contribuent au résultat global de l'apprenant au Learning Game (via un score, des badges, etc.). Cette structure est modulaire: elle permet l'ajout d'activités par les enseignants soit à la conception, soit lors des phases de réingénierie.

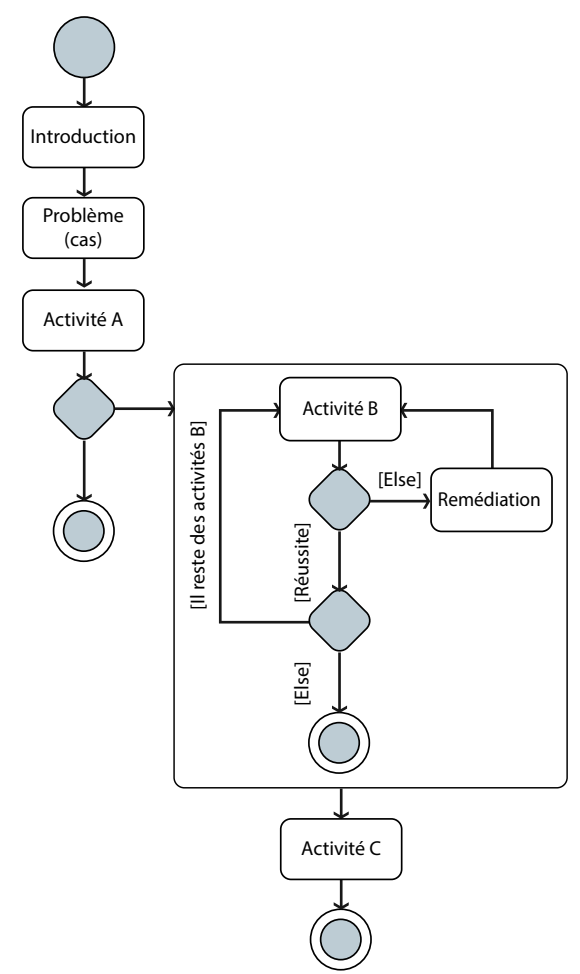

Figure $3 \cdot$ Modèle de graphe des scénarios $S_{i, j}$

\subsection{I, les activités et interactions}

Les activités peuvent être de différents types. Certaines mènent l'apprenant à opérer des choix ou à donner des résultats ou des informations par exemple en répondant à des questions. Les activités sont placées dans quatre catégories, sur la base des travaux de Carvalho (Carvalho et al., 2015) autour du modèle ATMSG (Activity Theory-based Model for Serious Games) : 
- ludique, cette catégorie regroupe les activités en lien avec la notion de jeu (par ex. récupérer un badge);

- d'apprentissage, les activités de cette catégorie permettent de travailler ou de vérifier des compétences, du point de vue de l'apprenantjoueur (par ex. réaliser un quiz);

- pédagogique intrinsèque, les activités de cette catégorie permettent de travailler ou de vérifier des compétences, mais sont placées du point de vue de l'enseignant et sont intrinsèques au jeu (donner un feedback automatique);

- pédagogique extrinsèque, les activités de cette catégorie permettent de travailler ou de vérifier des compétences mais sont placées du point de vue de l'enseignant et sont extrinsèques au jeu (communication entre apprenants sur le contenu du Learning Game via un forum).

Carvalho propose une décomposition de ces activités en actions portées par des outils et ayant un objectif spécifique associé aux catégories ci-dessus. Nous avons repris les taxonomies proposées par le modèle ATMSG largement inspirées par la littérature, telle la taxonomie de Bloom pour les objectifs pédagogiques (Anderson et al., 2001). Par exemple, la taxonomie proposée par Carvalho pour les actions ludiques est utilisée, entre autres, pour définir l'élément I.

L'ensemble I des interactions disponibles est défini par l'ingénieur pédagogique et proposé à l'enseignant. Chaque activité est associée par ce dernier à une ou plusieurs interactions disponibles dans l'ensemble I. Comme évoqué précédemment, nous avons utilisé les taxonomies d'actions proposées par Carvalho, assurant ainsi un large panel de possibilités pour les enseignants. Cet élément et l'ensemble des activités disponibles ne sont pas détaillés dans cet article.

\section{4. $C$, le contexte d'usage de $L_{g}$}

Le contexte d'usage $\mathrm{C}$ explicite les usages de $\mathrm{L}_{\mathrm{g}}$ et les contraintes associées à celui-ci. On intégrera par exemple l'environnement dans lequel les apprenants-joueurs se trouveront lors de l'utilisation de $\mathrm{L}_{g}$. Formellement, $\mathrm{C}$ est défini comme le quadruplet $\mathrm{C}=$ (Institutionnel, Spatial, Temporel, Matériel), avec:

- Institutionnel contient l'ensemble des contraintes propres au contexte institutionnel (type de cursus, type de formation, etc.);

- Spatial regroupe les contraintes de l'environnement spatial relatif aux usages de $\mathrm{L}_{\mathrm{g}}$ (salle de classe, extérieur, etc.) ; 
- Temporel reprend les éléments relatifs au temps (durée, fréquence, etc.) ;

- Matériel contient les contraintes matérielles (support, interface, etc.).

Plus précisément, l'élément $\mathrm{C}$ donne une description du cadre pédagogique dans lequel le Learning Game prend place et s'intègre. Il est défini par l'enseignant seul et tient compte des objectifs généraux du jeu définis au tout début de la phase de conception.

\subsection{Méthode associée au modèle DISC}

Comme dans le modèle DISCO, les éléments sont co-conçus par le ou les enseignants auteurs du Learning Game. Ces derniers sont présents dans toutes les phases de la conception (figure 4). Dans nos expérimentations, ils sont accompagnés par un ingénieur pédagogique qui facilite la production des documents relatifs aux éléments de DISC et prend en charge la phase de production du jeu.

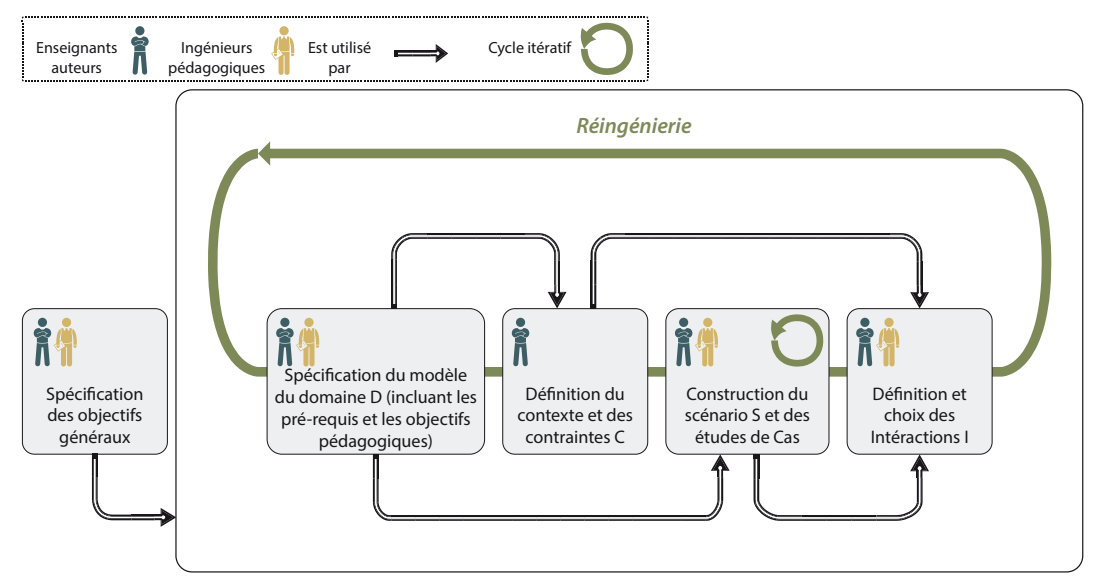

Figure 4 • Schéma indiquant les phases de la méthode associée au modèle DISC

La méthode associée à DISC est itérative, grâce à l'intégration d'un cycle de réingénierie. À l'issue de la co-conception d'un Learning Game, de son utilisation et d'une phase d'analyse des traces d'usage, une phase de réingénierie est opérée par les enseignants (auteurs et/ou utilisateurs). Le processus propose les phases suivantes: spécification des objectifs généraux, spécification de $\mathrm{D}$, définition de $\mathrm{C}$, co-construction de $\mathrm{S}$, définition et choix de I (figure 4). 
Le modèle DISC et la méthode associée ont été décrits à l'enseignant souhaitant concevoir un Learning Game en mécanique des fluides.

\subsection{Passation du modèle DISC}

Plusieurs passations avec cinq enseignants ont été réalisées entre avril et juin 2016 lors d'entretiens semi-directifs (tels que préconisés par la méthode THEDRE). Ces enseignants sont chargés de cours dans différentes écoles d'ingénieurs en France. Ils avaient pour objectif de réaliser des Learning Games en vue de permettre aux étudiants de manipuler des compétences propres à chaque discipline. Le modèle a été présenté à l'aide de documents, repris dans ce manuscrit, présentant les éléments et la structure de DISC. Nous avons explicité la méthode associée (figure 3), les documents attendus à produire et les outils utilisés, tels les outils de carte mentale.

L'un des enseignants, en charge de l'enseignement de la mécanique des fluides à l'IMT Lille Douai, a souhaité travailler avec ce modèle à la suite de l'entretien d'avril 2016. Lors d'un second entretien semi-directif, cet enseignant a précisé le type de traces à recueillir afin d'analyser les parcours des apprenants et, le cas échéant, de réaliser une réingénierie du jeu. Il a souhaité recueillir :

- les scores finaux des apprenants, mais aussi le nombre de points de vie en fin de jeu ;

- les pas visités ordonnés chronologiquement, ce qui permet de déduire le chemin des apprenants au sein du scénario $S$;

- le temps total passé sur le jeu ;

- le temps passé pour chaque pas ;

- le nombre de parties réalisées par chaque apprenant.

Nous reviendrons sur le recueil de traces des apprenants lors de la présentation de l'expérimentation.

5. Expérimentation : développement du Learning Game «Missions à Emosson 》

Cette partie décrit l'expérimentation menée pour valider le modèle DISC. Celle-ci a été planifiée à l'aide des outils proposés par THEDRE et cette planification est donnée par un diagramme d'orchestration (cf. figure 5).

L'enseignant auteur a conçu un Learning Game comme une activité du MOOC Introduction à la mécanique des fluides. Il souhaitait apporter aux 
apprenants une activité fondée sur une situation réelle mobilisant et faisant travailler les compétences vues lors du MOOC. Ce dernier a été utilisé au sein d'un dispositif de classe inversée pour les élèves ingénieurs de trois écoles d'ingénieurs françaises. Le Learning Game fait référence au complexe d'Emosson en Suisse non loin du massif du Mont Blanc. L'apprenant-joueur devra vivre le quotidien d'un ingénieur mécanicien des fluides en poste à Emosson S.A. et résoudre les problèmes et situations qui lui seront proposés (voir figure 6). Missions à Emosson (http://lesecsper.imt-lille-douai.fr/ECSPER_MaE/index.html) a été intégré au MOOC lors de sa deuxième session de septembre à novembre 2016 sur six semaines: deux semaines de travail suivies d'une semaine d'évaluation, puis deux semaines de travail pour terminer sur une semaine d'évaluation.

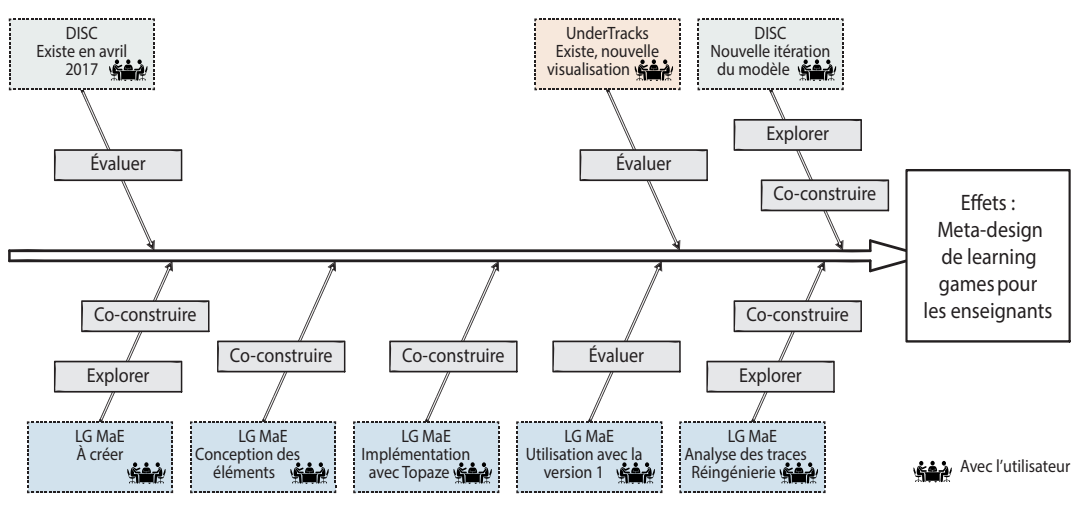

Figure 5 - Diagramme d'orchestration des outils activables DISC et du learning game Missions à Emosson.

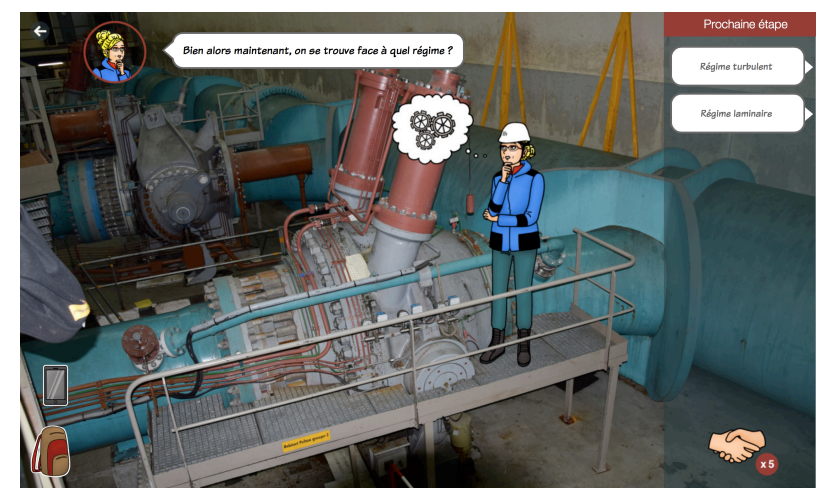

Figure 6 • Capture d'écran du learning Game Missions à Emosson. 


\subsection{Définition du modèle de domaine, des objectifs et du contexte}

La conception du Learning Game Missions à Emosson (MaE) a débuté en avril 2016. Une présentation du modèle DISC a été réalisée sous forme d'un entretien avec l'enseignant initiateur du projet. Cet entretien a permis de spécifier les livrables issus du modèle DISC attendus pour réaliser le Learning Game. Par la suite, l'enseignant a conçu le graphe du modèle du domaine $\mathrm{D}$ avec le soutien d'un ingénieur pédagogique. Nous avons construit ce graphe autour de quatre étapes $\mathrm{E}_{1}, \mathrm{E}_{2}, \mathrm{E}_{3}$ et $\mathrm{E}_{4}$, ces dernières étant relatives aux quatre chapitres du MOOC correspondant aux quatre semaines de travail. Par exemple, $\mathrm{E}_{2}$ a les mêmes prérequis et objectifs que le chapitre dynamique des fluides réels. Ce dernier point rend l'exercice aisé pour l'enseignant auteur et facilite la compréhension du concept de modèle du domaine. De fait, il a été alors très simple de définir les prérequis $P_{i}$ et les objectifs pédagogiques $O_{i}$ de chaque étape. Voyons un exemple illustrant cet aspect: l'étape $E_{2}$ est fondée sur le domaine $\mathrm{D}_{2}$ contenant 14 compétences (dont neuf prérequis) et 18 liens entre elles. $\mathrm{E}_{2}$ contient quatre études de cas indépendantes.

La structuration des $\mathrm{Ec}_{\mathrm{i}, \mathrm{j}}$ a immédiatement suivi en fonction du graphe D. Les prérequis et objectifs étant définis pour chaque étape, l'enseignant a construit les études de cas en respectant l'acronyme P.I.S.T.E. (voir 3.2) à partir de situations réelles, des prérequis $P_{\mathrm{i}}$ et des objectifs $\mathrm{O}_{\mathrm{i}}$. Le contexte $\mathrm{C}$ a été spécifié en parallèle : l'activité est intégrée à un MOOC grand public, suivi simultanément par des étudiants de trois écoles d'ingénieur.

\subsection{Conception du scénario et choix des interactions}

Le modèle des études de cas a permis la création de huit missions, chacune étant une étude de cas, correspondant aux quatre étapes: deux missions pour $E_{1}$, quatre pour $E_{2}$, une pour $E_{3}$ et une pour $E_{4}$ (figure 7). L'enseignant a proposé ces étapes en fonction du découpage en chapitre utilisé dans son cours. Cette hiérarchie des étapes a été construite lors de la conception du modèle du domaine $\mathrm{D}$. Une étape $\mathrm{E}_{\mathrm{i}}$ est accessible si l'apprenant possède les prérequis $P_{i}$ de $E_{i}$. Les compétences de $P_{i}$ sont travaillées dans une étape $E_{j}$ avec $i>j$.

Le graphe type des $S_{i, j}$ a rendu la création des scénarios des $\mathrm{Ec}_{\mathrm{i}, \mathrm{j}}$ plus aisée et le travail de conception plus efficace. L'enseignant a été partiellement autonome sur cette phase, un appui sporadique de 
l'ingénieur pédagogique a été nécessaire pour intégrer des éléments ludiques. Les interactions ont été choisies par l'enseignant en fonction du scénario des études de cas et des objectifs de chacune d'entre elles. Une première réalisation a permis de spécifier ces interactions ou de les modifier avec l'enseignant.

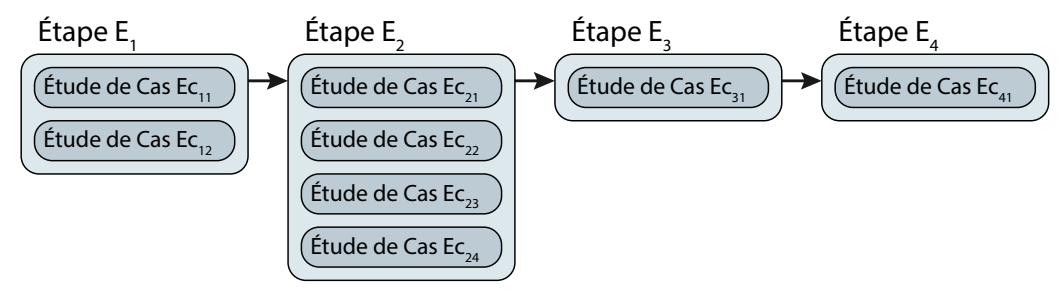

Figure 7 - Étapes et études de cas de MaE.

Le Learning Game a été réalisé avec Topaze, un modèle documentaire pour la chaine éditoriale Scenari (Quelennec et al., 2010). Ce modèle, conçu pour la création d'études de cas non linéaires numériques, est adaptable au modèle DISC et en particulier au modèle des $S_{i, j}$. La conception et le développement ont été étalés sur cinq mois et ont mobilisé quatre personnes : un enseignant, un ingénieur pédagogique et technique, une dessinatrice et un doctorant.

\subsection{Première évaluation du modèle DISC}

Nous avons organisé un entretien semi-directif avec l'enseignant auteur du Learning Game immédiatement après cette première expérimentation sur la base d'un guide d'entretien (entretien mené le 28 novembre 2016). Il s'agit d'un professeur de physique de 60 ans, peu à l'aise avec les outils informatiques, sensibilisé aux questions de pédagogie, mais novice en matière de Learning Game. Plusieurs éléments notables sont ressortis de cette rencontre.

L'enseignant a pointé les difficultés à définir le modèle du domaine a priori. Il souligne l'intérêt du fractionnement de cet élément et le rapprochement fait avec les chapitres du MOOC: «[...] J'ai associé les étapes avec les différents chapitres de cours, ce qui a facilité la création de celles-ci. Cela a aussi facilité la mise en place d'une progression adaptée dans la difficulté des étapes. » 
De plus, la notion d'étape lui semble intéressante pour la création des études de cas intégrant une progression au fur et à mesure de l'avancée dans le Learning Game. Le passage des exercices classiques de mécanique des fluides (avec application de connaissances) aux études de cas mobilisant des compétences a été délicat, mais revient à passer à une pédagogie par projet. La création des scénarios des études de cas a été facilitée par la présence de l'ingénieur pédagogique (ce point a été rappelé de nombreuses fois) et par l'usage du modèle de scénario avec les différents types d'activités. D'après l'enseignant, l'indépendance des missions (études de cas) a grandement facilité la conception de ces dernières en isolant les scénarios les uns des autres. De nouvelles missions sont d'ailleurs en gestation (en particulier pour les étapes $\mathrm{E}_{3}$ et $\mathrm{E}_{4}$ ).

Enfin la définition claire des missions de l'enseignant dans la conception a été appréciée: «Je savais ce que j'avais à faire, en particulier les documents à produire pour chaque élément [...], mais la collaboration avec Gaëlle [l'ingénieure pédagogique] était indispensable tout au long de la conception.»

Cette première évaluation est donc globalement positive. Pour autant, d'autres tests doivent être conduits pour valider ces premiers résultats avec d'autres enseignants.

\section{L'enseignant dans la phase d'usage}

Comme évoqué précédemment, Fischer fait intervenir deux temps dans le Meta-Design: la phase de conception et la phase d'usage. Le modèle DISC concerne principalement la phase de conception. Néanmoins, il a été conçu pour faciliter l'analyse des traces d'usage des étudiants par les enseignants, afin de leur permettre une réingénierie des Learning Games. Marne (Marne, 2014) a montré l'importance de la réingénierie des Serious Games par les enseignants eux-mêmes pour permettre leur adoption par ces derniers. Choquet (Choquet, 2007) définit la réingénierie pédagogique d'un EIAH (et donc d'un Learning Game) comme «l'examen d'une situation pédagogique médiatisée et la modification du dispositif d'apprentissage (l'EIAH proprement dit, mais aussi son contexte, tel que le rôle des acteurs, le contexte d'usage, les possibilités d'observation de l'activité) afin de reconstituer ce dernier sous une nouvelle forme et mettre ainsi en place une nouvelle situation pédagogique, prenant mieux en compte les usages observés et l'évolution des pratiques pédagogiques.» 
La réingénierie suppose donc une analyse globale de l'EIAH pour mettre en place une démarche d'amélioration de celui-ci à partir des données collectées auprès des acteurs gravitant autour de cet EIAH. Cette analyse impose au préalable le recueil de ces données issues des usages de l'EIAH par les différents acteurs (Choquet et Iksal, 2007).

\subsection{Analyse de traces d'usage des étudiants}

Dans notre cas, les retours d'usage sont des fichiers de données et de logs qui décrivent le chemin pris par les étudiants au travers des graphes du scénario. Nous avons analysé ces données avec les enseignants (auteur et utilisateurs) dans le but de comparer le chemin attendu par les enseignants et le chemin pris par les étudiants (nous utiliserons les termes de "scénario attendu » et "scénario observé » (Pernin et Lejeune, 2004). Ce chemin reprend la structure du Learning Game conçu avec DISC, permettant ainsi d'analyser le chemin de l'étudiant au sein des études de cas $\mathrm{Ec}_{\mathrm{i}, \mathrm{j}}$, mais aussi à l'échelle des étapes (figure 8).

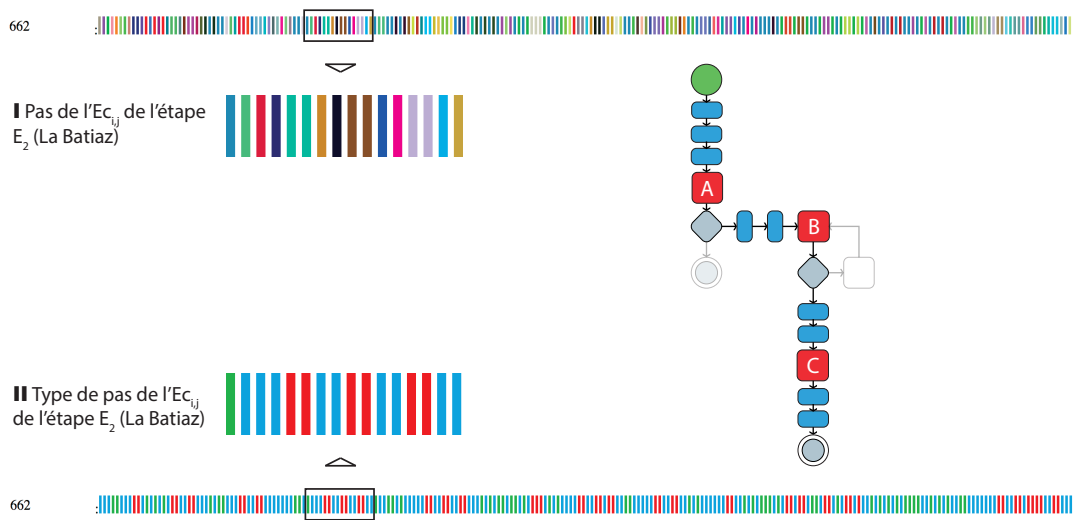

Figure 8 - Visualisations avec Undertracks du chemin de I'étudiant 662 avec focus sur l'étude de cas « La Batiaz» et du scénario associé.

De précédents travaux avaient permis de repérer au sein des chemins des étudiants des séquences de pas fréquentes menant à des erreurs, pour un Learning Game différent conçu comme une activité d'un MOOC en statistique (Vermeulen et al., 2016). L'analyse, menée au printemps 2017 sur un échantillon de 872 chemins distincts pour 609 étudiants uniques de l'ESTP (École Spéciale des Travaux Publics, une école d'ingénieur), a été 
réalisée par les enseignants avec l'objectif d'évaluer la difficulté du Learning Game Missions à Emosson et les potentiels points de blocage pour les étudiants. Étant donné le nombre important d'activités nécessaires pour réaliser le jeu, une analyse manuelle par les enseignants est trop complexe et demande trop d'efforts et de temps.

Ainsi, pour faciliter le travail des enseignants nous avons opté pour deux types de visualisations, toutes deux réalisées à l'aide de la plateforme Undertracks (Mandran et al., 2015), un outil conçu par le laboratoire informatique de Grenoble (LIG) pour capitaliser et analyser les données d'usage (http://undertracks.imag.fr). La première donne une visualisation des pas ordonnés chronologiquement, une couleur donnée étant associée à un pas unique (cf. figure 8, I en haut). La seconde affiche les mêmes pas dans le même ordre, mais la couleur correspond au type de pas (cf. figure 8 , II en bas):

- en vert, le pas correspondant au choix de la mission dans une étape donnée (pas de navigation);

- en bleu, les pas expositoires (introduction, exposé du cas dans les $\mathrm{S}_{\mathrm{i}, \mathrm{j}}$ pas narratifs ou de contenu le cas échéant);

- en rouge, les pas correspondant aux interactions dans les activités des $\mathrm{S}_{\mathrm{i}, \mathrm{j}}$ (de type A, B ou C).

Ces visualisations ont été mises en relation avec les données chiffrées brutes: temps passé, scores, etc. Ces éléments ont été proposés aux enseignants lors d'un entretien libre, afin de recueillir leurs propres éléments d'analyse.

\subsection{Résultat de I'analyse avec les enseignants et choix pour la réingénierie}

Un premier entretien a été mené fin août 2017 avec l'enseignant auteur du Learning Game Missions à Emosson. Ce dernier a très vite exprimé les limites de l'exercice face au grand nombre de données disponibles. Néanmoins il a pointé plusieurs éléments intéressants :

- 1) le temps de réalisation avec succès anormalement court pour un certain nombre d'étudiants, parfois largement inférieur à quatre heures (la durée de jeu a été estimée entre quatre et huit heures);

- 2) le nombre important d'étudiants ayant consulté les supports de cours durant de jeu (détecté à l'aide de la première visualisation) ;

- 3) la quasi-impossibilité de sortir du jeu avec un Game-Over; 
- 4) le grand nombre d'étudiants ayant réalisé au moins deux fois une des huit études de cas (détecté à l'aide de la seconde visualisation), avec un meilleur résultat dans les tentatives suivantes.

L'enseignant a fait plusieurs hypothèses pour expliquer ces observations, hypothèses appuyées par les autres enseignants utilisateurs du Learning Game. Pour expliquer le temps très court (point 1), les enseignants avancent un partage des réponses et des bons choix entre étudiants. Cette hypothèse a été validée après le Debriefing (ou séance de verbalisation) avec les étudiants qui a suivi l'utilisation du jeu (Lederman, 1992). Les étudiants ont mis en avant leur impatience à découvrir les illustrations et le scénario du jeu. Le second point semble montrer l'intérêt d'intégrer ces supports de cours au jeu: l'apprenant reste dans le jeu, mais peut consulter des ressources utiles pour sa progression dans le scénario. Le point 3 est expliqué par un trop grand nombre de récompenses en point de confiance (l'équivalent des points de vie) et le trop faible nombre de pertes de ces points, rendant le jeu trop facile. Enfin le quatrième point semble renforcer d'une part l'intérêt du choix des études de cas à réaliser et, d'autre part, celui du scénario non linéaire.

À la suite de ces observations, les enseignants ont fait des propositions pour améliorer le Learning Game, menant ainsi à une réingénierie de Missions à Emosson. Le point 1 a surtout influencé l'intégration du jeu au sein du dispositif pédagogique. Les enseignants ont intégré le jeu comme fil conducteur des travaux pratiques donnés aux étudiants, ces derniers réalisant les études de cas en présentiel, et comme activités libres pour les autres apprenants du MOOC. Le point 3 a amené à supprimer une partie des récompenses de point de confiance et à modifier le calcul des scores dans le jeu. L'enseignant propose par ailleurs une réflexion autour du Debriefing mis en place à l'issue de l'utilisation du jeu pour permettre d'une part l'explication des résultats obtenus par les étudiants, et d'autre part la prise de conscience des compétences acquises tout au long du Learning Game. La nouvelle version de Mission à Emosson a été intégrée à la troisième session du MOOC.

\section{Conclusion et perspectives}

À la suite de l'étape de réingénierie, la nouvelle version du Learning Game Missions à Emosson a été déployée pour la troisième session du MOOC Introduction à la mécanique des fluides en septembre 2017. Plus de 1100 élèves ingénieurs ont utilisé le jeu soit comme une activité d'auto- 
évaluation, soit comme support aux travaux dirigés dans un dispositif de classe inversée. Sept enseignants ont suivi cet usage et ont participé à une nouvelle analyse des traces d'usage. Cette analyse favorisera une nouvelle étape de réingénierie du Learning Game. Le travail mené et présenté dans cet article, ainsi que cette nouvelle phase d'usage mèneront à l'amélioration du modèle DISC, en travaillant à nouveau les éléments constitutifs de celui-ci, en particulier l'explicitation des types d'activités. Nous réaliserons ainsi un nouveau cycle de recherche selon la méthode THEDRE.

Cette amélioration se fera aussi au travers de la conception d'autres Learning Games avec d'autres enseignants, dans les mois à venir, en utilisant d'autres outils de développement. En particulier, la conception d'un Learning Game baptisé Smellville en chimie environnementale a débuté en mai 2017 avec un groupe de quatre enseignants. L'analyse des traces d'usage des apprenants étant un exercice difficile pour les enseignants, nous poursuivrons le travail sur la visualisation de ces traces par et pour ce public avec Undertracks. Il s'agit par exemple d'identifier automatiquement les études de cas au sein du chemin pris par les étudiants et de proposer une nouvelle visualisation aux enseignants. Une autre piste est d'utiliser des algorithmes de fouille de données pour repérer des séquences de pas fréquentes (Guerra et al., 2014) au sein des chemins des apprenants pour identifier des comportements intéressants: erreurs fréquentes, sorties du jeu avant la fin de celui-ci, etc.

D’une manière générale, le travail présenté marque une étape vers le Meta-Design des Learning Games. En dehors de ces derniers, le Meta-Design est une approche intéressante qui, bien que définie initialement par Fischer pour la conception de logiciels, pourrait être étendue à d'autres domaines. Les travaux décrits ici peuvent mener à la mise en place d'une approche Meta-Design des activités ou dispositifs pédagogiques avec ou sans usage du numérique. Cette approche peut aussi favoriser la montée en compétences des enseignants, en les impliquant fortement dans l'analyse des usages des outils et dispositifs qu'ils conçoivent, et même, par la facilitation de la collaboration multidisciplinaire, l'émergence d'innovations pédagogiques.

\section{REFERENCES}

Alvarez, J., Plantec, J.-Y., Vermeulen, M. et Kolski, C. (2017). RDU Model dedicated to evaluate needed counsels for Serious Game projects. Computers \& Education, 114, 38-56. https://doi.org/10.1016/j.compedu.2017.06.007 
Anderson, L. W., Krathwohl, D. R., Airasian, P., Cruikshank, K., Mayer, R., Pintrich, P., Raths, J., Merlin C. et Wittrock, M. (2001). A taxonomy for learning, teaching and assessing: A revision of Bloom's taxonomy. New York, NY : Longman.

Avenier, M.-J. (2009). A methodological framework for constructing generic knowledge with intended value both for academia and practice (Cahiers de recherche, n 2009-01 E4). CERAG, Université de Grenoble. Récupéré de l'Archive HAL: https://halshs.archives-ouvertes.fr/halshs-00526616/document

Bowker, G. C. et Star, S. L. (2000). Sorting things out: Classification and its consequences. MIT press.

Carvalho, M. B., Bellotti, F., Berta, R., De Gloria, A., Sedano, C. I., Hauge, J. B., JU, J. et Rauterberg, M. (2015). An activity theory-based model for serious games analysis and conceptual design. Computers \& Education, 87, 166- 181. https://doi.org/10.1016/j.compedu.2015.03.023

Choquet, C. (2007). Ingénierie et réingénierie des EIAH - L'approche REDiM (Mémoire HDR, Université du Maine, Le Mans, France). Récupéré de l'Archive HAL : https://hal.archives-ouvertes.fr/tel-00276225/

Choquet, C. et Iksal, S. (2007). Modélisation et construction de traces d'utilisation d'une activité d'apprentissage: une approche langage pour la réingénierie d'un EIAH. Sciences et technologies de l'information et de la communication pour l'éducation et la formation (STICEF), 14. Récupéré du site de la revue :http://sticef.univ-lemans.fr/num/vol2007/14choquet/sticef_2007_choquet_14p.pdf

Cohn, M. (2004). User stories applied: For agile software development. AddisonWesley Professional.

Daniau, S. (2005). Jeu de Rôle Formatif et maturation des adultes - Co-rechercheaction-formation et approche écobiopsychosociale (Thèse de doctorat, Université Paul Valéry-Montpellier III). Récupéré de TEL: https://tel.archives-ouvertes.fr/tel00167629/

Egenfeldt-Nielsen, S. (2004). Practical barriers in using educational computer games. On the Horizon, 12(1), 18-21.

Fabricatore, C. (2000). Learning and videogames: An unexploited synergy. Communication présentée au Workshop In Search of the Meaning of Learning, Annual Convention of the Association for Educational Communications and Technology (AECT), Long Beach, CA. Récupéré de : http://eprints.hud.ac.uk/28000.

Fischer, G., Giaccardi, E., Ye, Y., Sutcliffe, A. G. et Mehandjiev, N. (2004). Metadesign: a manifesto for end-user development. Communications of the ACM, 47(9), 33-37.

Guerra, J., Sahebi, S., Lin, Y.-R. et Brusilovsky, P. (2014). The problem solving genome: Analyzing sequential patterns of student work with parameterized exercises. Dans Proceedings of the $7^{\text {th }}$ International Conference on Educational Data Mining (EDM 2014) (p.153-160). Récupéré de http://educationaldatamining.org/EDM2014/uploads/procs2014/long\%20papers/1 53_EDM-2014-Full.pdf

Lederman, L. C. (1992). Debriefing: Toward a systematic assessment of theory and practice. Simulation \& Gaming, 23(2), 145-160. https://doi.org/10.1177/1046878192232003 
Mandran, N. et Dupuy-Chessa, S. (2017). THEDRE: A Traceable process for high quality in human centred computer science research. Dans N. Paspallis, M. Raspopoulos, C. Barry, M. Lang, H. Linger et C. Schneider (dir.), Proceedings of the $26^{\text {th }}$ International Conference on Information Systems Development (ISD 2017). Larnaca: University of Central Lancashire Cyprus.

Mandran, N., Ortega, M., Luengo, V. et Bouhineau, D. (2015). DOP8: Merging both data and analysis operators life cycles for technology enhanced learning. Dans Proceedings of the Fifth International Conference on Learning Analytics and Knowledge (LAK 2015) (p. 213-217). New York, NY : ACM.

Marchiori, E. J., Torrente, J., del Blanco, Á., Moreno-Ger, P., Sancho, P. et Fernández-Manjón, B. (2012). A narrative metaphor to facilitate educational game

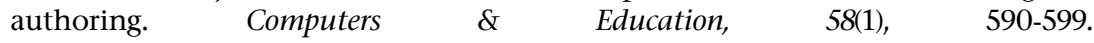
https://doi.org/10.1016/j.compedu.2011.09.017

Marfisi-Schottman, I. (2012). Méthodologie, modèles et outils pour la conception de Learning Games (thèse de doctorat, INSA de Lyon, France). Récupéré de TEL : https://tel.archives-ouvertes.fr/tel-00762855/

Marfisi-Schottman, I., Labat, J.-M. et Carron, T. (2013). Approche basée sur la méthode pédagogique des cas pour créer des Learning Games pertinents dans de nombreux domaines d'enseignement. Dans Actes de la conférence Environnements Informatiques pour l'Apprentissage Humain (EIAH'2013) (p.67-78). Récupéré de https://www.irit.fr/EIAH2013/uploads/ActesEIAH2013.pdf

Mariais, C., Michau, F., Pernin, J.-P. et Mandran, N. (2011). Learning Role-Playing Games: méthodologie et formalisme de description pour l'assistance à la conception. Premiers résultats d'expérimentation. Dans Actes de la conférence Environnements Informatiques pour l'Apprentissage Humain (EIAH'2011) (p. 95-107). Mons, Belgique: UMons. Récupéré de Hal: https://hal.archives-ouvertes.fr/hal00605892/file/Mariais-Christelle-EIAH2011.pdf

Marne, B. (2014). Modèles et outils pour la conception de jeux sérieux : une approche meta-design (thèse de doctorat, Université Pierre et Marie Curie - Paris VI, France). Récupéré de TEL : https://tel.archives-ouvertes.fr/tel-01134701/

Marne, B. et Labat, J.-M. (2014). Model and authoring tool to help teachers adapt serious games to their educational contexts. International Journal of Learning Technology, 9(2), 161-180.

Marne, B., Wisdom, J., Huynh-Kim-Bang, B. et Labat, J.-M. (2012). The six facets of serious game design: A methodology enhanced by our design pattern library. Dans Proceedings of the 21st Century Learning for 21st Century Skills (EC-TEL 2012) (p. 208-221).

Melero, J., El-Kechaï, N. et Labat, J.-M. (2015). Comparing two CbKST approaches for adapting learning paths in serious games. Dans Design for Teaching and Learning in a Networked World, Proceedings of the 10th European Conference on Technology Enhanced Learning (EC-TEL 2015) (p. 211-224). Cham, Suisse : Springer.

Pernin, J.-P. et Lejeune, A. (2004). Dispositifs d'apprentissage instrumentés par les technologies: vers une ingénierie centrée sur les scénarios. Dans Actes du colloque Technologies de l'Information et de la Connaissance dans l'Enseignement Supérieur et dans l'Industrie (TICE 2004) (p. 407-414).

Quelennec, K., Vermeulen, M., Narce, C. et Baillon, F. (2010). De l'industrialisation à l'innovation pédagogique avec une chaîne éditoriale. Dans Actes $d u 7^{i m e}$ colloque Technologies de l'Information et de la Communication pour 
l'Enseignement (TICE 2010). Récupéré de HAL : https://hal.archives-ouvertes.fr/hal01169404/document

Reimann, P., Kickmeier-Rust, M. et Albert, D. (2013). Problem solving learning environments and assessment: A knowledge space theory approach. Computers \& Education, 64, 183-193. https://doi.org/10.1016/j.compedu.2012.11.024

Rowe, J. P., Shores, L. R., Mott, B. W. et Lester, J. C. (2011). Integrating learning, problem solving, and engagement in narrative-centered learning environments. International Journal of Artificial Intelligence in Education, 21(1-2), 115-133.

Sanchez, E., Emin-Martinez, V. et Mandran, N. (2015). Jeu-game, jeu-play, vers une modélisation du jeu. Une étude empirique à partir des traces numériques d'interaction du jeu Tamagocours. Sciences et technologies de l'information et de la communication pour l'éducation et la formation (STICEF), 22(1), 9-44.

Sokovic, M., Pavletic, D. et Pipan, K. K. (2010). Quality improvement methodologies-PDCA cycle, RADAR matrix, DMAIC and DFSS. Journal of Achievements in Materials and Manufacturing Engineering, 43(1), 476-483.

Vermeulen, M. (2016). Un modèle formel de jeux sérieux de type étude de cas pour l'enseignement supérieur: le modèle DISCO. Dans Actes des $6^{e}$ Rencontres Jeunes Chercheurs en EIAH (RJC'EIAH 2016) (p.119-124). Récupéré de HAL: https://hal.archives-ouvertes.fr/hal-01322650/document

Vermeulen, M., Fleury, A., Fronton, K. et Laval, J. (2015). Les ALPES : Approches agiLes Pour l'Enseignement Supérieur. Dans Actes $d u$ ème Colloque Questions de Pédagogie pour l'Enseignement Supérieur (QPES 2015) (p. 243-247).

Vermeulen, M., Laval, J., Serpaggi, X. et Pinot, R. (2017). Soyez agiles dans les A.L.P.E.S.! Une pédagogie en mode agile. Dans Actes du 9ème Colloque Questions de Pédagogie dans l'Enseignement Supérieur (QPES 2017) (p. 845-852). Récupéré de HAL : https://hal.archives-ouvertes.fr/hal-01503723/document

Vermeulen, M., Mandran, N. et Labat, J.-M. (2016). Chronicle of a scenario graph: From expected to observed learning path. Dans Proceedings of 11th European Conference on Technology Enhanced Learning (EC-TEL 2016) (p.321-330). Springer International. https://doi.org/10.1007/978-3-319-45153-4_24

Vygotsky, L. S. (1980). Mind in society: The development of higher psychological processes. Harvard university press.

Yusoff, A., Crowder, R., Gilbert, L. et Wills, G. (2009). A Conceptual Framework for Serious Games. Dans Proceedings of Ninth IEEE International Conference on Advanced Learning Technologies (ICALT 2009) (p.21-23). https://doi.org/10.1109/ICALT.2009.19 